\title{
Barcoding success as a function of phylogenetic relatedness in Viburnum, a clade of woody angiosperms
}

\author{
Wendy L Clement ${ }^{*}$ and Michael J Donoghue
}

\begin{abstract}
Background: The chloroplast genes matK and rbcL have been proposed as a "core" DNA barcode for identifying plant species. Published estimates of successful species identification using these loci (70-80\%) may be inflated because they may have involved comparisons among distantly related species within target genera. To assess the ability of the proposed two-locus barcode to discriminate closely related species, we carried out a hierarchically structured set of comparisons within Viburnum, a clade of woody angiosperms containing ca. 170 species (some 70 of which are currently used in horticulture). For 112 Viburnum species, we evaluated $r b c \mathrm{~L}+$ matK, as well as the chloroplast regions rp/32-trnL, trnH-psbA, trnK, and the nuclear ribosomal internal transcribed spacer region (nrlTS).

Results: At most, rbcL + matK could discriminate $53 \%$ of all Viburnum species, with only $18 \%$ of the comparisons having genetic distances $>1 \%$. When comparisons were progressively restricted to species within major Viburnum subclades, there was a significant decrease in both the discriminatory power and the genetic distances. trnH-psbA and nrlTS show much higher levels of variation and potential discriminatory power, and their use in plant barcoding should be reconsidered. As barcoding has often been used to discriminate species within local areas, we also compared Viburnum species within two regions, Japan and Mexico and Central America. Greater success in discriminating among the Japanese species reflects the deeper evolutionary history of Viburnum in that area, as compared to the recent radiation of a single clade into the mountains of Latin America.

Conclusions: We found very low levels of discrimination among closely related species of Viburnum, and low levels of variation in the proposed barcoding loci may limit success within other clades of long-lived woody plants. Inclusion of the supplementary barcodes trnH-psbA and nrlTS increased discrimination rates but were often more effective alone rather than in combination with $r b c L+$ matK. We surmise that the efficacy of barcoding in plants has often been overestimated because of the lack of comparisons among closely related species. Phylogenetic information must be incorporated to properly evaluate relatedness in assessing the utility of barcoding loci.
\end{abstract}

\section{Background}

The use of a short fragment of DNA sequence to distinguish between species -- DNA barcoding -- promises to streamline species identification, thereby enabling scientific research (e.g., studies of community ecology) and practical applications (e.g., monitoring the movement of biological materials across borders). The ideal DNA barcode would be a single locus that could be universally amplified and sequenced for a broad range of taxa, be easily aligned over large phylogenetic distances, and

\footnotetext{
* Correspondence: wendy.clement@yale.edu

Department of Ecology and Evolutionary Biology, Yale University, P.O. Box 208105, New Haven, CT 06520, USA
}

\section{Biomed Central

(c) 2012 Clement and Donoghue; licensee BioMed Central Ltd. This is an Open Access article distributed under the terms of the Creative Commons Attribution License (http://creativecommons.org/licenses/by/2.0), which permits unrestricted use, distribution, and reproduction in any medium, provided the original work is properly cited. provide sufficient variation to reliably distinguish closely related species. The zoological community has adopted cytochrome oxidase I (COI) as a DNA barcode that appears to generally fulfill these criteria. In contrast, the plant community has struggled to identify a single marker with these qualities $[1,2]$ and botanists have favored the use of a multilocus barcode [3-5]. Specifically, the Plant Working Group of the Consortium for Barcodes of Life has proposed the combined use of short segments of the chloroplast genes mat $\mathrm{K}$ and $r b c \mathrm{~L}$ as a "core" plant barcode [5]. However, in view of the fact that mat $\mathrm{K}$ and $r b c \mathrm{~L}$ have not been considered the best choices in a number of individual studies $([2,6-9]$ but see 
also $[10,11])$, the use of supplementary, typically more variable barcodes, such as trn $\mathrm{H}-p s b \mathrm{~A}$ and the nuclear ribosomal internal transcribed spacer regions (nrITS), has been suggested as a means of increasing the efficacy of the $r b c \mathrm{~L}+$ mat K barcode [12].

In the search for a plant barcode, universality and ease of amplification and sequencing have been prioritized $[4,5,13]$, and these criteria played a major role in the choice of $r b c \mathrm{~L}+$ mat $\mathrm{K}$ [5]. The discriminatory power of $r b c \mathrm{~L}+m a t \mathrm{~K}$ has been evaluated in a number of studies, but the effects of taxon sampling in such studies requires further analysis. In several studies that have presented comparisons that widely span the angiosperms, it has been calculated that $r b c \mathrm{~L}+$ matK are able to distinguish $70-80 \%$ of the species [3-5,14]. As a proxy for comparing closely related species, some of these studies have included two or more species from within a number of plant genera, but phylogenetic trees were not specifically used to gauge the relatedness of the species sampled. This is problematical. For example, when placed in a phylogenetic context (Figure 1), the five species of the genus Viburnum (Adoxaceae, Dipsacales) that have been included in such comparisons $[4,5]$ turn out to represent widely separated clades that have been diverging from one another for tens of millions of years. Comparing only these species may overestimate the ability to distinguish among closely related species using the proposed markers. Generally, because genera come in many sizes and ages, the random sampling of selected species within a genus does not ensure that these species are actually very closely related to one another. Direct phylogenetic information is necessary to determine how closely or distantly related the species are.

The success of barcoding also depends on the analytical methods employed. So-called character-based approaches [16] can differentiate plant species based on one or a few variable base pairs, while more commonly used methods based on genetic distances (e.g., using a predetermined cut-off of 1\%) or tree-based approaches may require greater amounts of genetic variation [9]. Here too, it is important to test such methods on species whose relatedness has been inferred phylogenetically. To establish meaningful barcoding guidelines and standards, it ultimately will be essential to carry out comparisons of both markers and analytical methods within a welldefined phylogenetic framework.

Some barcoding applications, such as inventories of biodiversity hotspots [17], require the differentiation of species only within a given geographic area, and comparisons within regions have generally reported higher species discrimination rates using plant barcodes $([12,18,19]$, but see [20). For example, Kress et al. [18] were able to discriminate $98 \%$ of the species in barcoding the plants on Barro Colorado Island in Panama; the only problems were within genera with more than one species on the Island, such as Ficus, Inga, and Piper. Such results may reflect a general pattern, namely that very closely related plant species seldom grow sympatrically. However, as some evolutionary circumstances can yield such sympatry (e.g., polyploidy speciation), the efficacy of community-level or regional barcoding efforts also needs to be evaluated in a phylogenetic context. In general, we would expect better discrimination when the several members of particular genera within an area represent relatively distantly related clades.

Here we evaluate the discriminatory power of potential plant barcodes within the context of a phylogeny for the woody flowering plant clade Viburnum (Adoxaceae, Dipsacales). This clade contains approximately 170 species (Figure 1) and is of great interest to the horticultural community as more than 70 of these species (and various artificial hybrids) are currently in cultivation ([21]; Figure 1). The ability to distinguish closely related $\mathrm{Vi}$ burnum species using barcodes would be extremely useful in identifying horticultural material and in monitoring the movement of these economically important plants (as cuttings or seeds) around the globe.

Viburnum naturally occupies the temperate regions of North America and Eurasia and extends into the montane forests of Latin America and into tropical habitats in Southeast Asia. Most Viburnum species are diploids with $2 \mathrm{~N}=18$ [22,23]. Homoploid speciation has been postulated in a few specific instances [24-26], though evidence for this is still limited. Allopolyploidy appears to have occurred several times [23,25]. The New World Oreinodontotinus clade is characterized by chromosome numbers of 36 and, occasionally, of 72 [22,23,27]. An aneuploidy reduction to $2 \mathrm{~N}=16$ characterizes the Asian Solenotinus clade, within which chromosome numbers of 32 and 64 are also found [23]. Hybridization is possible between members of the different section-level clades [22], but it is not especially common in the wild, and hybrid swarms and introgression have seldom been documented and are associated with recent human disturbance [27].

Although the species-level taxonomy of Viburnum is currently under review, many steps have recently been taken to confirm the number of species that exist in the wild. Approximately 894 Viburnum species names appear in IPNI (www.ipni.org), Tropicos (www.tropicos. org) and The Plant List (www.theplantlist.org). More than a decade ago, Malécot and Donoghue (unpublished) reduced this list to 229 recognized species (the remaining names being placed in synonymy). In light of recent regional studies and other ongoing assessments, this list has been further refined and we now recognize ca. 170 species (Figure 1). Additionally, a series of recent phylogenetic studies has confidently identified the major clades within Viburnum and their relationships to one 


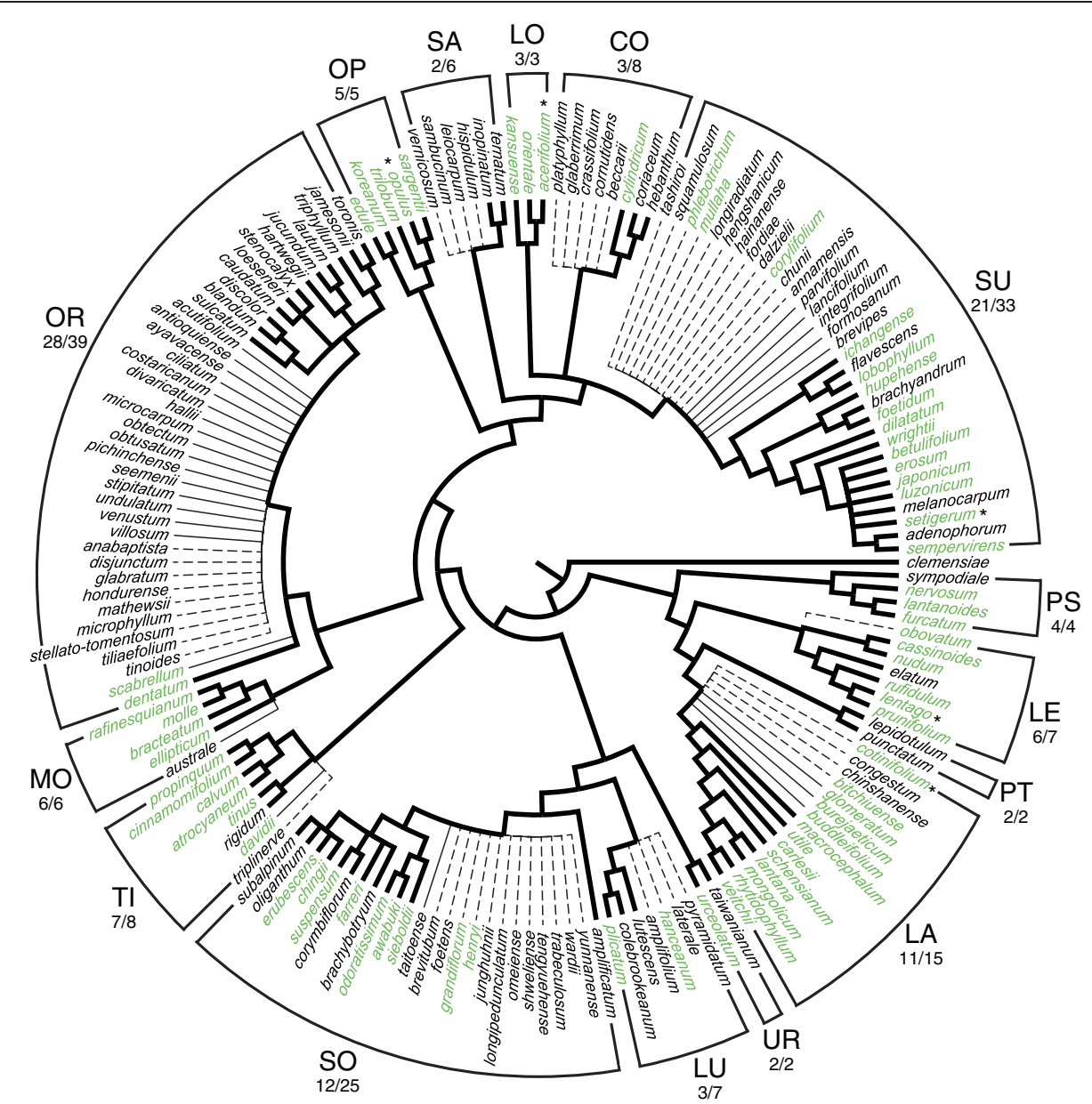

Figure 1 Phylogenetic representation of the 170 currently recognized species of Viburnum. Branches in bold represent the 90 species and evolutionary relationships recovered in Clement and Donoghue [15]. Thin branches are those species added to the study of Viburnum in this paper, and dashed branches are Viburnum species that have yet to be sampled. These species are placed at the base of the named clade to which they are expected to belong based on previous taxonomic and morphological studies. Species names in green are those that are currently used in the horticultural industry, and species marked by an asterisk were sampled in Fazekas et al. [4] and in CBOL Plant Working Group [5]. Informal clade names (following [15]), and the number species sampled in this study followed by the total number of species assigned to the clade, are provided. Abbreviations are as follow: CO-Coriacea, LA-Lantana, LE-Lentago, LO-Lobata, LU-Lutescentia, MO-Mollodontotinus, OP-Opulus, OR-Oreinodontotinus, PS-Pseudotinus, PT-Punctata, SA-Sambucina, SO-Solenotinus, SU-Succodontotinus, TI-Tinus, UR-Urceolatum.

another [15,25,26,28]. These studies provide a solid framework within which to evaluate the power of barcode markers and methods to discriminate species globally, or within particular geographic regions, as a function of their degree of relatedness. Specifically, we focus on a set of hierarchically structured comparisons within Viburnum using the $r b c \mathrm{~L}+$ matK core barcode, as well as three other chloroplast markers $(r p l 32-t r n \mathrm{~L}$, $\operatorname{trn\mathrm {K}}$, and $t r n \mathrm{H}-p s b \mathrm{~A}$ ) and the nrITS region. trn $\mathrm{H}-p s b \mathrm{~A}$ was once a contender as the plant DNA barcode $[3,5,29]$, and the utility of ITS2 has recently been highlighted as an alternative to $r b c \mathrm{~L}+\operatorname{matK}$ [30-32]. In addition to making comparisons within and across all of Viburnum, we also evaluate the performance of these markers in a regional context, focusing especially on
Viburnum species within Japan and within Mexico and Central America.

\section{Methods}

\section{Species sampling}

We obtained sequences from all of the 90 species used in our most recent phylogenetic study [15], with the exception of $V$. lepidotulum, from which we were able to obtain too few sequences. To this sample we added data for 28 previously unsequenced Viburnum species. As explained below, we lumped several pairs of previously separated species so as not to underestimate the discriminatory power of the plant barcodes. In total, we analyzed 112 species, 40 of which were represented by two to six individuals. Material for the newly acquired 
accessions was obtained from herbarium specimens from the Harvard University Herbaria ( $\mathrm{HUH})$, the Field Museum (F), the Missouri Botanical Garden (MO), the New York Botanical Garden (NY), and our own collections in silica gel with corresponding voucher specimens in the Yale University Herbarium (YU). Voucher information and Genbank accession numbers are provided in Additional file 1.

As they were in part designed to test the relationships of proposed "segregate" species, our previous phylogenetic studies included representatives of several potential Viburnum species that are not presently considered to be distinct in recent regional taxonomic treatments. For present purposes we wanted to reduce the number of species in these cases so as not to bias the barcoding results by artificially reducing genetic distances. Specifically, we lumped $V$. awabuki with $V$. odoratissimum [33], V. calvum with $V$. atrocyaneum [34], V. scabrellum with $V$. dentatum [35], $V$. taiwanianum with $V$. urceolatum [33], and $V$. veitchii with $V$. glomeratum [34]. In several instances, however, we did not reduce species complexes as proposed in some regional floras based on our own conflicting geographic or molecular evidence. Thus, we maintained $V$. australe and $V$. affine as distinct from $V$. rafinesquianum on the basis of their geographic ranges. Also, in view of the results of Clement and Donoghue [15], we treated $V$. adenophorum, $V$. flavescens, $V$. hupehense, and $V$. lobophyllum as distinct from $V$. betulifolium (contra [34]). Similarly, we recognized $V$. bracteatum as distinct from $V$. molle, and $V$. cylindricum as distinct from $V$. coriaceum.

\section{DNA extraction and data collection}

Total genomic DNA was extracted from herbarium and silica dried specimens using a Qiagen DNeasy kit (Valencia, CA). The initial step of the extraction protocol was modified for herbarium tissue by adding Bmercaptoethanol and proteinase $\mathrm{K}$ to ground leaf tissue and shaken for $12-24$ hours at $42^{\circ} \mathrm{C}$ [36].

Amplification and sequencing protocols for matK, trnH-psbA, rpl32-trnL, trnK, and nrITS followed Clement and Donoghue [15]. The barcoding region of $r b c \mathrm{~L}$ was obtained from previously sequenced taxa by truncating the sequences to match the proposed barcoding region. In instances where we were unable to sequence the entire $r b c \mathrm{~L}$ gene region, we followed the $r b c \mathrm{~L}$ barcoding protocol [5] using $r b c$ La_f [3] and $r b c$ La_rev [5] primers.

PCR products were sequenced in forward and reverse directions using the amplification primers at either the DNA Analysis Facility on Science Hill or the Keck DNA Sequencing Facility at Yale University. Sequences were assembled using Sequencher 4.10.1 (Gene Codes Corp.) and aligned using Muscle 3.6 [37]. Gene region alignments were manually reviewed and edited.

\section{Phylogenetic analysis}

With 28 species new to the study of the Viburnum phylogeny, we conducted a phylogenetic analysis including one representative of all 112 species and the six genes examined in this study (Additional file 1). The data were separated into two partitions, one containing all chloroplast gene regions and the second containing nrITS. Models for each partition were selected using MrModeltest [38]. Phylogenetic analyses were performed with MrBayes v3.1.2 [39], with 30 million generations using six chains, sampling the posterior distribution every 1,000 generations. Plots of the likelihood and model parameters were examined in Tracer 1.5 [40] to assess convergence and determine an appropriate burnin.

\section{Barcode evaluation and species identification}

We evaluated six candidate plant barcoding markers, including five chloroplast regions and nrITS. First, each gene region was evaluated independently. Then, we concatenated and evaluated $r b c \mathrm{~L}$ and $m a t \mathrm{~K}$ together, as this is the core plant DNA barcode proposed by the CBOL Plant Working Group [5]. Lastly, we concatenated a third gene region (supplementary barcode) to this core barcode. Specifically, we evaluated the discriminatory power of $r b c \mathrm{~L}+m a t \mathrm{~K}+t r n \mathrm{H}-p s b \mathrm{~A}$ and of $r b c \mathrm{~L}+m a t \mathrm{~K}$ + nrITS. Because the number of accessions per species varied, calculations involving interspecific comparisons were obtained from a data set that included only one representative accession per species (Additional file 1). Intraspecific comparisons were made separately.

We evaluated potential barcodes in three ways. First, we identified the number of unique sequences (i.e., haplotypes) within each data set using TCS [41], which provided an absolute maximum number of species that could be identified with the data. With this approach, successful discrimination of two species could entail a difference of just one base pair. Then, the number of unique sequences was divided by the number of species included in the dataset to obtain an estimate of the maximum percentage of species that could be discriminated by the data. Second, we calculated genetic distances under a Kimura 2 parameter (K2P) model using PAUP $4 \mathrm{~b} 10$ [42] for both intra- and interspecific comparisons. We did not include the same number of accessions per species, and not all species were represented by more than one accession. To control for this, we averaged the intraspecific variation within each species to prevent artificially increasing or decreasing the overall levels of interspecific variation detected in the data. Histograms were compiled using $\mathrm{R}$ version 2.13.0 [43] to examine the variation in the data and to compare intra- and interspecific genetic distances. Third, using the genetic distances generated from pair wise comparisons among all species in the data set, we report the percentage of 
comparisons with genetic distances that exceed 1\% and $2 \%$. We recognize that any such cutoff is arbitrary, but these cutoffs appear commonly in the literature and allows a comparison to results from less inclusive clades of Viburnum as described below.

\section{Hierarchical evaluation of barcode performance}

To explore the discriminatory power of barcoding regions in an evolutionary framework, we used our $\mathrm{Vi}$ burnum phylogeny to inform a set of comparisons. Specifically, we focused on the four largest named clades within Viburnum: Lantana, Oreinodontotinus, Solenotinus, and Succodontotinus [15,26]. We compiled the data described above for each of these four clades separately: 11 of the 15 species of Lantana, 28/ 39 species of Oreinodontotinus, 12/ 25 species of Solenotinus, and 21/ $\sim 33$ species of Succodontotinus.

\section{Barcode evaluation using regional samples}

To explore the discriminatory power of the various barcodes within more restricted geographical areas, we focused on two regions: Japan and Mexico and Central America. Our data include 14 of the 16 species described from Japan [33], and all 17 species described from Mexico and Central America [27]. We compiled the standard nine datasets for each of the two geographical regions and analyzed the data as described above.

\section{Results}

\section{Discriminatory power across Viburnum}

Information on the number of species sampled, total aligned sequence length, and number of variable characters for each gene region and combination of gene regions is given in Table 1. The number of identical sequences in the datasets is also shown in Table 1. For this calculation, gaps were treated as missing data, so the differences between sequences were based only on point mutations (nucleotide substitutions). When gaps were coded as a $5^{\text {th }}$ state, the number of unique sequences increased for all gene regions except mat $\mathrm{K}$ and $r b c \mathrm{~L}$ (Table 1). However, using gaps as traits is difficult because the occurrence of gaps can change depending on taxon sampling; gaps could prove useful once all species of Viburnum have been properly sampled.

The number of identical sequences was used to calculate a maximum identification proportion (Max ID rate; Table 1 ). In this case, two species need differ by only a single base pair to be considered successfully differentiated. Applying this approach to the mat $\mathrm{K}$ and $r b c \mathrm{~L}$ data, we were only able to identify $39 \%$ and $19 \%$ of the species sampled, respectively, and just over $50 \%$ when the two regions were combined (Table 1). The other chloroplast regions sampled yielded slightly higher proportions ( 49-63\% of species differentiated). nrITS was the most variable gene region and by itself could discriminate $90 \%$ of the species sampled.

Intra- and interspecific genetic distances were calculated as a second approach to evaluating discriminatory power (Figure 2; Tables 1 and 2). Mean interspecific genetic distances for mat $\mathrm{K}$ and $r b c \mathrm{~L}$ were 0.0087 and 0.0058 , respectively, and still less than $1 \%$ when combined. All of the other barcoding regions evaluated have mean genetic distances greater than $1 \%$ (Figure 2; Table 1). The mean intraspecific variation for each barcode was quite low with average comparisons for regions of $0.58 \%$ or less (Table 2). Even with our limited

Table 1 Viburnum interspecific comparisons for barcode gene regions

\begin{tabular}{|c|c|c|c|c|c|c|c|c|c|}
\hline \multirow[b]{2}{*}{ Gene Region } & \multirow[b]{2}{*}{ Species } & \multirow[b]{2}{*}{$\begin{array}{l}\text { Aligned } \\
\text { Length }\end{array}$} & \multirow[b]{2}{*}{$\begin{array}{l}\text { Variable } \\
\text { characters }\end{array}$} & \multirow[b]{2}{*}{$\begin{array}{l}\text { Unique } \\
\text { sequences }\end{array}$} & \multirow[b]{2}{*}{$\begin{array}{l}\text { \% Max } \\
\text { ID rate }\end{array}$} & \multicolumn{3}{|c|}{ K2P Genetic Distances } & \multirow[b]{2}{*}{$>2 \%$} \\
\hline & & & & & & Max & Mean (SD) & $>1 \%$ & \\
\hline matk & 98 & 725 & 62 & 38 & 38.78 & 0.0255 & $0.0087(0.0047)$ & 34.36 & 0.95 \\
\hline$r b c L$ & 103 & 491 & 18 & 20 & 19.42 & 0.0187 & $0.0058(0.0047)$ & 24.77 & 0 \\
\hline$r p / 32-t r n L$ & 97 & 942 & 119 & $61(70)$ & 62.89 & 0.0352 & $0.0152(0.0073)$ & 78.95 & 27.32 \\
\hline $\operatorname{trnH}-p s b \mathrm{~A}$ & 108 & 491 & 103 & $54(79)$ & 50.00 & 0.0597 & $0.0184(0.0098)$ & 84.94 & 41.64 \\
\hline $\operatorname{trnK}$ & 97 & 1068 & 90 & $48(52)$ & 49.48 & 0.0211 & $0.0108(0.0053)$ & 64.37 & 1.50 \\
\hline nrlTS & 105 & 628 & 202 & $94(97)$ & 89.52 & 0.1117 & $0.0528(0.02)$ & 94.63 & 89.80 \\
\hline$r b c L+$ matK & 94 & 1216 & 79 & 50 & 53.19 & 0.0184 & $0.0074(0.0033)$ & 18.23 & 0 \\
\hline $\begin{array}{l}r b c \mathrm{~L}+\text { matK } \\
+\operatorname{trnH}-p s b \mathrm{~A}\end{array}$ & 91 & 1707 & 175 & 67 & 73.63 & 0.0231 & $0.0100(0.0041)$ & 67.94 & 0.46 \\
\hline $\begin{array}{l}r b c L+\text { matK } \\
+ \text { nrlTS }\end{array}$ & 88 & 1844 & 261 & 86 & 97.73 & 0.0396 & 0.0219 (0.0078) & 88.71 & 72.52 \\
\hline
\end{tabular}

For each gene region, the number of species analyzed, the aligned length of the gene region, the number of variable characters, the number of unique sequences with gaps treated as missing data (number with gaps treated as a fifth state), and the maximum number of species that can be identified by the data (Max ID rate = Identical sequences/total number of species) are reported. Also provided are summary statistics of genetic distances using a Kimura 2-parameter (K2P) model and include: maximum genetic distance (max), mean interspecific distance (mean) with standard deviation (SD), and the proportion of comparisons of genetic distances greater than $1 \%(>1 \%)$ and greater than $2 \%(>2 \%)$. See Additional file 1 for details on species sampling. 

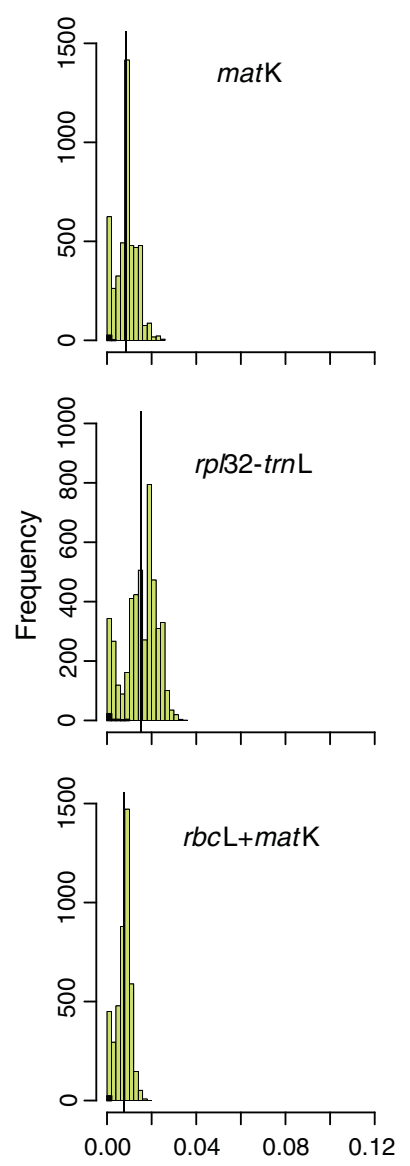
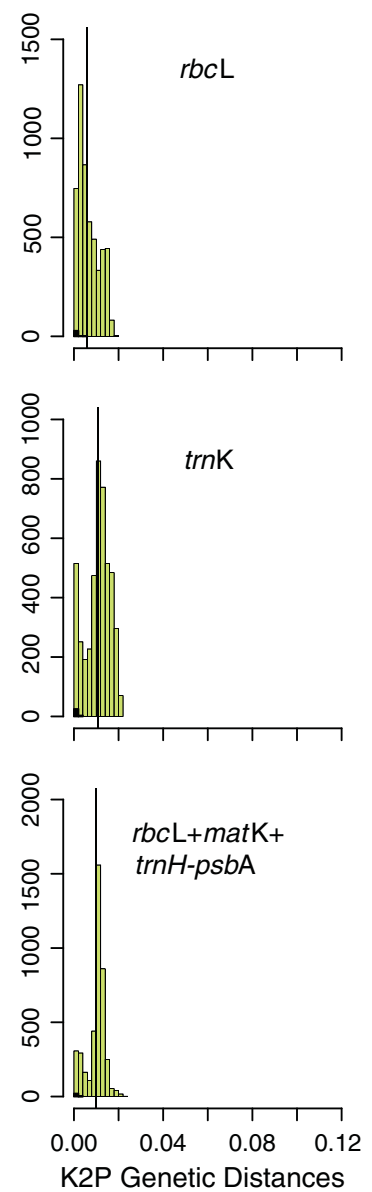
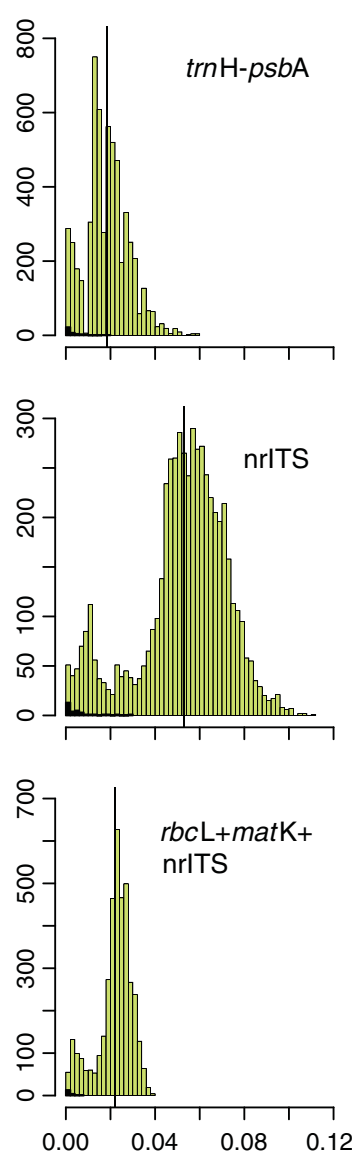

Figure 2 Intra- and interspecific genetic distances of barcoding genes across Viburnum. Histograms showing intraspecific (black bars) and interspecific (green bars) genetic distances calculated using a K2P model of sequence evolution for each gene region or combination of gene regions. Each histogram contains the name of the gene region or regions sampled and a black vertical line indicating the mean genetic distance for the gene region(s).

sampling of intraspecific variation, we observed complete overlap of the distributions of intraspecific and interspecific variation (Figure 2), so there was no natural "barcoding gap" [44] to use as a cut-off for distinguishing species. Minimum genetic distances for both intraand interspecific comparisons were zero, and for most gene regions there were a significant number of comparisons with a genetic distance of zero. In the absence of a clear gap, we calculated discriminatory power using $1 \%$ and $2 \%$ differences. At the level of $1 \%, r b c \mathrm{~L}+$ mat K distinguished $18 \%$ of the species; less than $1 \%$ of species comparisons differed by more than $2 \%$ (Table 1 ). This indicates that the majority of the unique sequences identified differed at very few nucleotide sites.

\section{Subclade analyses}

The Bayesian analysis of all six genes sampled in this study (Figure 3) recovered all of the major clades identified in Clement and Donoghue [15] with the exception that here the three species of Lobata do not form a clade
(Figure 3). In some instances, support for previously recognized clades was diminished, but this is likely due to the reduction in the genes sampling: six genes and $4,345 \mathrm{bp}$ as compared to ten genes and 9,552 bp in Clement and Donoghue [15].

As expected, comparisons within the Lantana, Oreinodontotinus, Solenotinus, and Succodontotinus clades (Figure 3) showed a significant decrease in the level of genetic variation relative to comparisons made across all of Viburnum (Figure 4). For each gene region or combination of regions, the genetic variation decreased by more than 50\% (Figure 4; Additional file 2). With the exception of nrITS alone and $r b c \mathrm{~L}+$ matK + nrITS, none of the mean genetic distances exceeded 1\% (Figure 4).

\section{Regional comparisons}

Mean genetic distances among the Mexican and Central American species were very low (Table 3) and similar to results for the Oreinodontotinus clade that includes all but two of the species from this region ( $V$. elatum of the 
Table 2 Viburnum intraspecific comparisons based on Kimura 2-Parameter (K2P) genetic distances

\begin{tabular}{lllll}
\hline Gene Region & Accessions & Species & Max & Mean (SD) \\
\hline matK & 75 & 26 & 0.0028 & $0.0002(0.0006)$ \\
rbcL & 92 & 31 & 0.0041 & $0.0004(0.0009)$ \\
rp/32-trnL & 94 & 32 & 0.0084 & $0.0020(0.0027)$ \\
trnH-psbA & 109 & 39 & 0.0187 & $0.0029(0.0043)$ \\
trnK & 82 & 28 & 0.0038 & $0.0008(0.0011)$ \\
nrlTS & 90 & 32 & 0.0295 & $0.0058(0.0073)$ \\
rbcL + matK & 68 & 23 & 0.0017 & $0.0002(0.0004)$ \\
rbcL + matK + & 64 & 22 & 0.0025 & $0.0006(0.0008)$ \\
trnH-psbA & & 19 & 0.0075 & $0.0018(0.0019)$ \\
rbcL + matK + & 57 & & & \\
nrlTS & & & &
\end{tabular}

Lentago clade; $V$. australe of the Mollodontotinus clade). Using the proposed barcoding markers, a maximum of $40 \%$ of the species could be identified and the average genetic distance among these species was only $0.1 \%$. nrITS was the most variable locus, followed by $\operatorname{trn} \mathrm{H}-$ $p s b \mathrm{~A}$. In Japan, $r b c \mathrm{~L}+$ mat $\mathrm{K}$ discriminated many more species, and higher levels of genetic variation were observed for all of the markers (Table 3 ).

\section{Discussion}

We sampled approximately two thirds of all Viburnum species (112 of 170 species) and were able to distinguish at most $53 \%$ of the species sampled using the proposed plant barcode, $r b c \mathrm{~L}+m a t \mathrm{~K}$, and a character-based method that accepts even single base differences between species (Table 1). Similar upper estimates were calculated within four major clades within Viburnum (Figure 4; Additional file 2). However, estimates of species discrimination varied dramatically depending on the proportion of the Viburnum clade sampled and the method used to implement the barcode (see [9] for further discussion). When we used genetic distances the discrimination rate decreased to $18 \%$ (Table 1 ). Within Viburnum subclades we found that none of the average genetic distances were greater than $1 \%$; that is, only one species could be recognized within each of these clades (Figure 4; Additional file 2). Overall, our findings based on the intensive sampling of a single group of plants yields far lower estimates of discriminatory power than the $70 \%$ reported in broader surveys using $r b c \mathrm{~L}+$ mat $\mathrm{K}$ that include fewer closely related species [5]. As noted above, this result in Viburnum does not appear to reflect prevalent hybridization or allopolyploidy.

Supplementary barcodes have been proposed as a means to improve the efficacy of $r b c \mathrm{~L}+$ matK in discriminating closely related species, especially in groups with low levels of genetic variation [3-5]. We evaluated four additional markers and applied the two most variable, trnH-psbA and nrITS, as supplementary barcodes, and this yielded some improvement in discrimination. Using a character-based method, we could differentiate up to $98 \%$ of Viburnum with rbcL + matK + nrITS (Table 1). Discrimination rates using genetic distances were consistently lower $(0 \%$ at the $2 \%$ level with $r b c \mathrm{~L}+$ $m a t \mathrm{~K})$, and improvement based on the addition of supplementary barcodes depended on the gene region $(0.46 \%$ and $73 \%$ at the $2 \%$ level with $r b c \mathrm{~L}+$ mat $\mathrm{K}+$ nrITS and $r b c \mathrm{~L}+m a t \mathrm{~K}+t r n \mathrm{H}-p s b \mathrm{~A}$, respectively; Table 1).

Our findings highlight four major points discussed below: (1) for some plant groups, $r b c \mathrm{~L}+$ matK will not be variable enough to differentiate closely related species; (2) estimates of the discriminatory power of the $r b c \mathrm{~L}+$ mat $\mathrm{K}$ barcode have been overestimated by not including demonstrably closely related species; (3) discriminatory success on a regional level depends on the particular representation of subclades within genera within an area; and (4) phylogenetic trees provide the necessary framework for evaluating the success of barcoding as a function of relatedness.

\section{$r b c \mathrm{~L}+$ matK rarely differentiate closely related Viburnum species}

Of the loci we sampled, matK and $r b c \mathrm{~L}$ were the least variable, and the least able to differentiate closely related species. All other loci examined had average genetic distances greater than 1\%; trn $\mathrm{H}$-psbA was the most variable chloroplast locus and nrITS was the most variable marker of those tested (Figure 4). trn $\mathrm{H}$-psbA was rejected as a core plant barcode because of difficulties in amplification and sequencing [5], and because inverted repeats may also be prevalent [45]. Potential problems with nrITS, including inconsistent amplification and incomplete concerted evolution, have been thoroughly discussed in opposition to the use of nrITS as a core barcode $[5,12,46]$. Recent work has revisited the use of nrITS, and more specifically ITS2 [30,32,47], due to its universality and ease of amplification from many types of preserved tissues (e.g., old herbarium specimens; processed plants in herbal medicines). Despite potential difficulties, trnH-psbA and nrITS can be very useful supplementary barcodes within some plant groups $[6,12,31,48-51]$, and this is certainly the case in Viburnum.

In future work it will be important to bear in mind potential interaction effects in combining more and less variable markers. Thus, in our case, the core + supplementary barcode was outperformed by the supplementary barcode alone. However, this result is sensitive to the method used to apply the barcode. In characterbased methods, adding more markers simply adds more 
information. In genetic distance approaches, adding highly variable markers to invariable markers dilutes the genetic distances, making species discrimination less likely. $t r n \mathrm{H}-p s b \mathrm{~A}$ and nrITS are useful as supplementary barcodes, but may actually be more effective when used alone in groups with slower rates of molecular evolution.

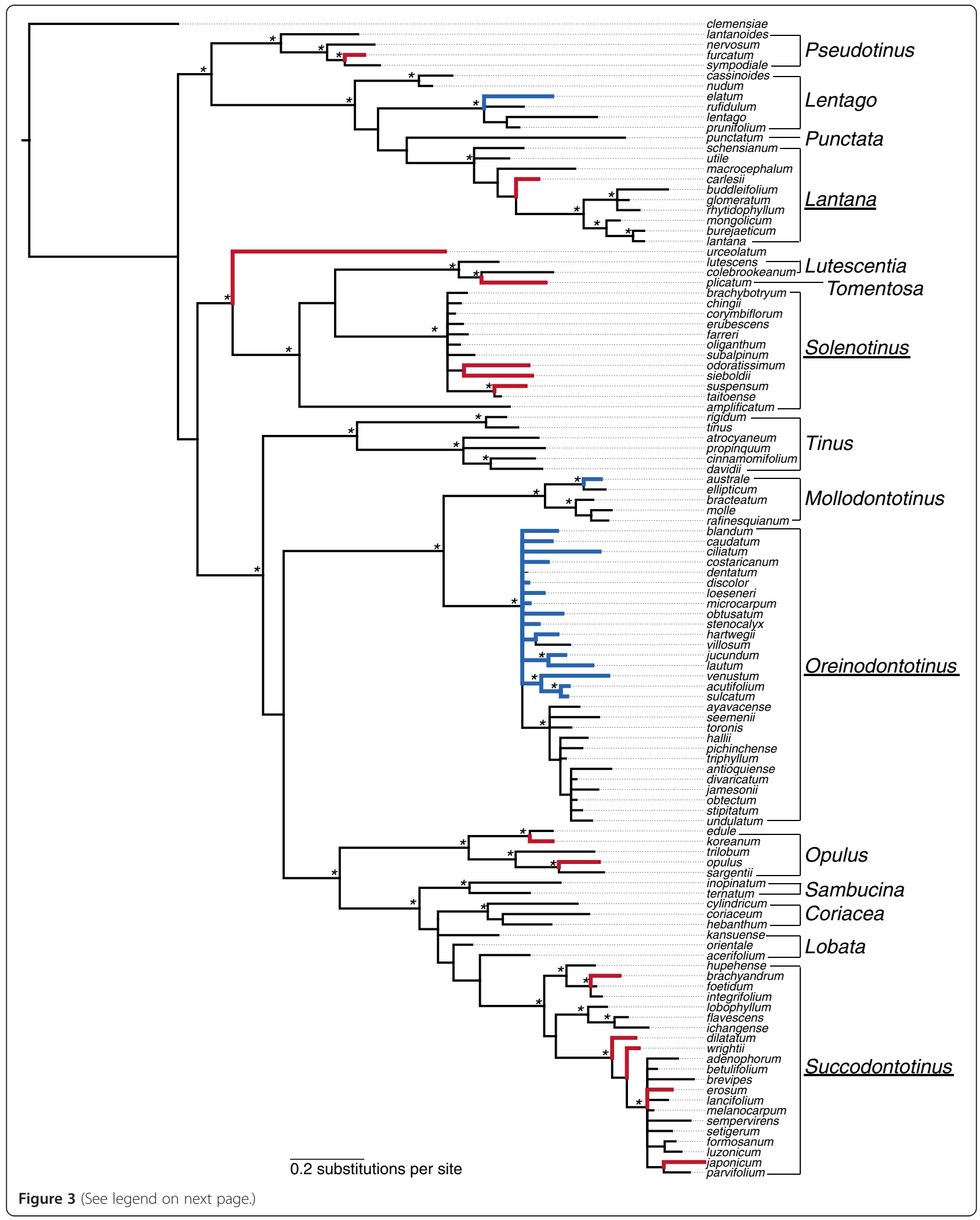


(See figure on previous page.)

Figure 3 Phylogeny of the $\mathbf{1 1 2}$ species of Viburnum sampled in this study. Presented is the Bayesian majority rule consensus tree of the combined chloroplast and nrlTS data. Asterisks above the branches indicate posterior probabilities greater than 0.95 . The named clades within Viburnum proposed by Winkworth and Donoghue [26] and Clement and Donoghue [15] are shown to the right, and the underlined clade names are those used in the hierarchical comparisons (see text). Thick red and blue branches mark Viburnum species that occur in Japan and in Mexico and Central America, respectively. The chloroplast and nrlTS partitions were each analyzed under a GTR $+1+\mathrm{G}$ model of sequence evolution. Resulting tree statistics and rate parameters are as follows: $-\mathrm{InL}=14052.08$; chloroplast partition - rate matrix $=0.2398,0.1810,0.0819,0.0903$, $0.1738,0.2331$, basepair frequencies $=0.3224,0.1643,0.1700,0.3435, G=0.0602, I=0.7412$; nrlTS partition - rate matrix $=0.0662,0.2027,0.0490$, $0.0341,0.6076,0.0405$, base pair frequencies $=0.1913,0.3203,0.2880,0.1989, G=0.7646, I=0.4152$.

Our findings suggest that for species identification purposes alone it may be an inefficient use of time and money to continue to sequence matK and $r b c \mathrm{~L}$ in groups where these markers show very little variation.

Viburnum plants are woody (shrubs and small trees) with relatively long generation times and slow rates of molecular evolution as compared to more rapidly evolving herbaceous lineages [52]. We believe that the limited variability seen in Viburnum will characterize many other groups of woody plants. Indeed, several studies of woody plant groups are consistent with this prediction regardless of the methods used to assess species discrimination. For example, $r b c \mathrm{~L}$ alone is unable to distinguish genera within Juglandaceae [50], and neither $r b c \mathrm{~L}$ nor mat $\mathrm{K}$ could discriminate species of Berberis, Ficus, or Gossypium [6]. Studies of Ligustrum (Oleaceae; [49]) and Alnus (Betulaceae; [48]) show that trnH-psbA and nrITS discriminated two to six times as many species as either

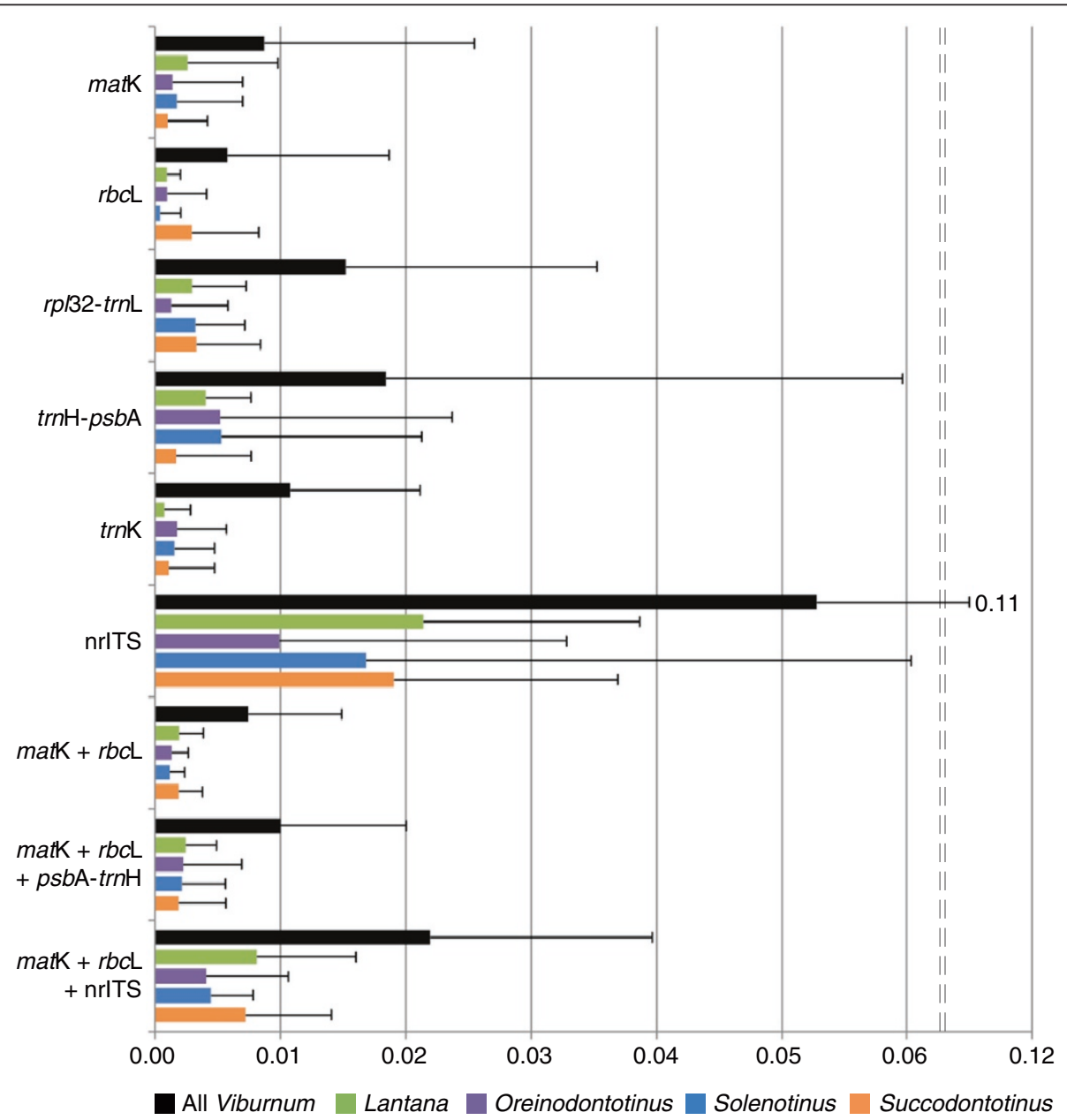

Figure 4 Genetic variation within Viburnum subclades. Bar graph showing the mean genetic distances calculated using all included Viburnum species and genetic distances calculated using the included species from four clades nested within Viburnum. The maximum genetic distance of each gene region or regions is indicated by a thin bar extending from the mean. 
Table 3 Summary of interspecific comparisons for regional Viburnum samples

\begin{tabular}{|c|c|c|c|c|c|c|c|c|c|}
\hline Gene Region & Species & $\begin{array}{l}\text { Aligned } \\
\text { Length }\end{array}$ & $\begin{array}{l}\text { Variable } \\
\text { characters }\end{array}$ & $\begin{array}{l}\text { Unique } \\
\text { sequences }\end{array}$ & $\begin{array}{l}\% \text { Max } \\
\text { ID rate }\end{array}$ & Max & Mean (SD) & $>1 \%$ & $>2 \%$ \\
\hline \multicolumn{10}{|c|}{ Central America and Mexico (17 species) } \\
\hline matK & 14 & 719 & 10 & 3 & 21.29 & 0.0126 & $0.0022(0.0044)$ & 14.29 & 0 \\
\hline$r b c L$ & 12 & 491 & 3 & 4 & 33.33 & 0.0041 & $0.0015(0.0018)$ & 0 & 0 \\
\hline$r p / 32-t r n L$ & 15 & 876 & 22 & 8 & 53.33 & 0.0208 & $0.0038(0.0057)$ & 13.33 & 0.95 \\
\hline $\operatorname{trnH}-p s b \mathrm{~A}$ & 16 & 410 & 17 & $10(12)$ & 62.50 & 0.0265 & $0.0084(0.0065)$ & 35.83 & 8.33 \\
\hline trnK & 12 & 1057 & 24 & 7 (8) & 58.33 & 0.0202 & $0.0047(0.0066)$ & 16.67 & 6.06 \\
\hline nrlTs & 17 & 602 & 77 & $14(15)$ & 82.35 & 0.0829 & $0.0213(0.0222)$ & 56.62 & 33.09 \\
\hline$r b c L+$ matK & 10 & 1210 & 5 & 4 & 40.00 & 0.0033 & $0.0010(0.0012)$ & 0 & 0 \\
\hline$r b c \mathrm{~L}+$ matK $+\operatorname{trn} \mathrm{H}-p s b \mathrm{~A}$ & 11 & 1612 & 15 & $7(9)$ & 63.63 & 0.0070 & $0.0025(0.0018)$ & 0 & 0 \\
\hline$r b c L+$ matK + nrlTs & 11 & 1807 & 61 & 10 & 91.00 & 0.0232 & $0.0068(0.0060)$ & 20.00 & 3.63 \\
\hline \multicolumn{10}{|l|}{ Japan (16 species) } \\
\hline matK & 13 & 725 & 24 & 10 & $76.92 \%$ & 0.0155 & $0.0081(0.0040)$ & 32.05 & 0 \\
\hline$r b c L$ & 13 & 491 & 8 & 6 & $46.15 \%$ & 0.0144 & $0.0065(0.0048)$ & 35.90 & 0 \\
\hline rp/32-trnL & 13 & 873 & 46 & 11 & $84.62 \%$ & 0.0266 & $0.0162(0.0073)$ & 76.92 & 35.90 \\
\hline $\operatorname{trnH}-p s b \mathrm{~A}$ & 13 & 439 & 41 & 10 & $76.92 \%$ & 0.0477 & $0.0176(0.0099)$ & 85.90 & 34.62 \\
\hline $\operatorname{trnK}$ & 14 & 1057 & 36 & 10 & $71.43 \%$ & 0.0172 & $0.0093(0.0043)$ & 48.35 & 0 \\
\hline nrlTS & 13 & 608 & 104 & 13 & $100.00 \%$ & 0.0914 & $0.0551(0.0213)$ & 97.44 & 89.74 \\
\hline$r b c L+$ matK & 13 & 1216 & 32 & 11 & $84.62 \%$ & 0.0125 & $0.0074(0.0032)$ & 12.82 & 0 \\
\hline$r b c \mathrm{~L}+$ matK $+\operatorname{trn} \mathrm{H}-p s b \mathrm{~A}$ & 12 & 1654 & 65 & 10 & $83.33 \%$ & 0.0157 & $0.0097(0.0041)$ & 71.21 & 0 \\
\hline$r b c \mathrm{~L}+$ matK + nrlTS & 12 & 1823 & 134 & 12 & $100.00 \%$ & 0.0372 & $0.0230(0.0089)$ & 86.36 & 69.70 \\
\hline
\end{tabular}

For the Viburnum species of Mexico and Central America and for Japan, the number of species analyzed, the aligned sequence length, the number of variable characters, the number of unique sequences with gaps treated as missing data (and with gaps treated as a fifth state), and the maximum number of species that can be identified by the data (Max ID rate = Identical sequences/total number of species) are reported. Summary statistics of genetic distances using a Kimura 2parameter (K2P) model include: maximum genetic distance (max), mean interspecific distance (mean) with standard deviation (SD), and the proportion of comparisons of genetic distances greater than $1 \%(>1 \%)$ and greater than $2 \%(>2 \%)$.

rbcL or matK. And in Quercus (which may have additional complications owing to hybridization) matK and $r b c \mathrm{~L}$ were unable to distinguish any of the 12 sympatric species examined [51]. And, among non-flowering woody plants, the $r b c \mathrm{~L}$ and matK barcode were not variable enough to differentiate Mexican cycads [53] or species of Picea [54]. The method of implementing barcodes is not uniform across these studies. However, the message is clear; levels of genetic variation in woody plants are low and barcoding is less successful. Character-based methods may make best use of little variation as these methods could potentially rely on as little as a single base pair [16]. However, it will be important to consider the minimum difference for species identification and to have proper intraspecific sampling to verify the consistency of DNA sequences within a species. Lastly, in woody plant groups where barcoding genes are reported to have higher rates of discrimination $[11,55]$, it would be interesting to establish the phylogenetic relatedness of the species sampled and to increase the species sampling to see if such results continue to hold.

\section{Insights from sampling closely related species}

Our study did not include enough replicates within species to critically compare levels of intra- and interspecific variation. However, given the very low genetic distances, we are confident that the inclusion of more accessions of each species would have very little effect. Instead, an increase in discriminatory power must await the development of more variable markers.

Importantly, we found many cases in which morphologically distinct and geographically separated species were genetically identical or nearly so. For instance, the Mexican species $V$. jucundum and $V$. acutifolium differ dramatically from one another in leaf and inflorescence size $[27,56]$, but are genetically identical according to $r b c \mathrm{~L}+$ matK. More specifically, $V$. jucundum plants are small trees with leaves averaging $11 \mathrm{~cm}$ in length and $9 \mathrm{~cm}$ in width, as compared to $V$. acutifolium plants, which are small shrubs with leaves which are typically $4 \mathrm{~cm}$ length and $2.5 \mathrm{~cm}$ width (i.e., $3 \mathrm{x}$ smaller). Genetic distances increased by $0.3 \%$ and $0.05 \%$ with the addition of trnH-psbA and nrITS, respectively. Similarly, within the Asian Succodontotinus clade, V. melanocarpum is 
readily distinguished by its distinctive black-colored fruits from all of its close relatives with red fruits, yet these species are nearly identical based on the available sequences. For example, the distances separating $V$. melanocarpum from $V$. dilatatum ranged from $0.17-0.55 \%$ depending on the supplementary barcode used.

This is not to say, however, that all of the species in our analysis can be easily distinguished based on morphology alone. Species boundaries in Viburnum are especially difficult in the Andes of South America (Figure 3; the clade containing $V$. ayavacense through $V$. undulatum; see [57]), where populations have been diverging from one another for only a short time [58,59]. Included in this clade are eight species from Ecuador that are genetically and morphologically quite similar. Although these species cannot be distinguished based on the barcodes examined here, our recent field studies confirm that these are distinct based a combination of one or more morphological characters, on microsatellite data, and on their geographic ranges (Donoghue, Sweeney, and Clement, MS in prep.).

\section{Species discrimination in a regional context}

Community-level or regional barcoding studies are becoming more common, and typically report higher species discrimination rates. In general, this reflects the fact that local floras are mainly comprised of distantly related species, typically representing many families and orders. Success in discriminating species within the genera with two or more species within an area will depend on how closely related these species are, which will vary depending upon speciation mechanisms and the biogeographic history of the group in question. We examined species discrimination in Viburnum in two broad regions, which yielded contrasting results.

Japanese Viburnum species represent six major clades (Lantana, Opulus, Pseudotinus, Solenotinus, Succodontotinus, and the isolated $V$. urceolatum), which have long been evolving separately ([15]; Figure 3). Not surprisingly, our discrimination success was quite high in this case. By comparison, 15 of the 17 Mexican and Central American Viburnum species are all members of a single major clade, Oreinodontotinus (Figure 3), and have radiated into the mountains of this region quite recently [58,59]. Understandably, our success in discriminating the species in this area was very low. The general message is that successful discrimination depends directly on the evolutionary and biogeographic history of the group in question, which can vary dramatically from one community or region to another.

\section{Conclusions}

Our study suggests that broad comparative studies of the success of the proposed plant barcodes have tended to overestimate the discriminatory power by failing to include a sufficient number of comparisons of very closely related species. In particular, the power of the $r b c \mathrm{~L}+$ mat $\mathrm{K}$ barcode is overrated. In Viburnum it is generally possible to confidently distinguish species belonging to the different major clades using the core barcodes, but the failure rate is very high when we consider close relatives within these clades. Even when we are able to differentiate species within these clades using a character-based approach (i.e., accepting any single nucleotide difference), genetic diversity is extremely low and methods based on genetic distances generally fail to distinguish close relatives even when these show clear-cut morphological and geographical differences. We suspect that similar results will be found in other plant groups, but especially in other woody plant groups with relatively long generation times and slow rates of molecular evolution [52]. Moving forward, we encourage the evaluation of the relative success of barcoding in an explicitly phylogenetic context, where the relative relatedness of the species being sampled can be established with confidence. To the extent that our findings are general, we also encourage the plant barcoding community to expand the multilocus barcode to include the additional markers necessary to accurately discriminate between closely related species. Although this may mean compromising somewhat on the ease of amplification and on universality, we believe that the benefits of being able to accurately identify a much higher proportion of species will be well worth the extra effort.

\section{Additional files}

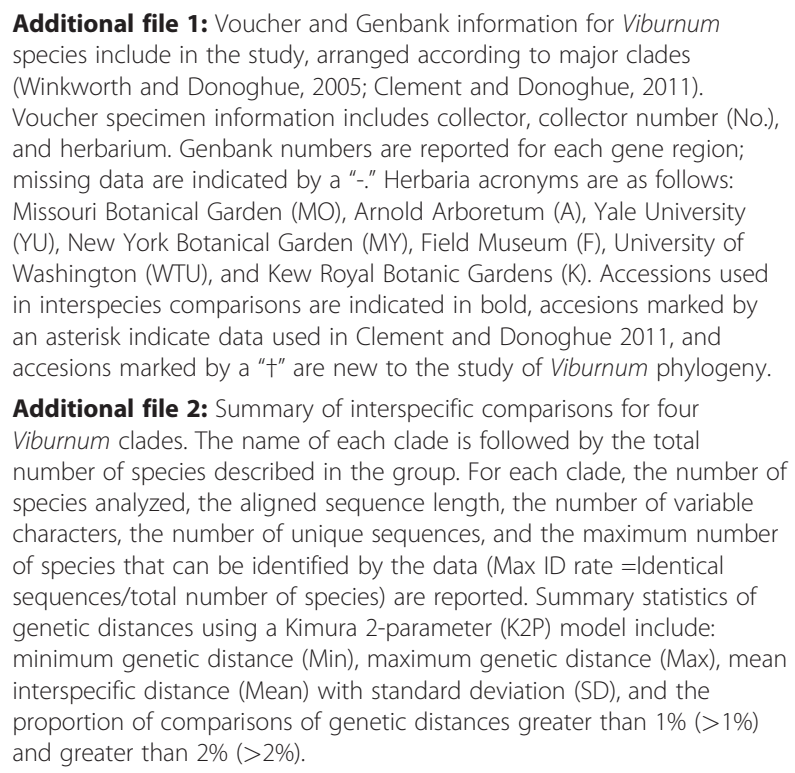




\section{Acknowledgements}

We thank the following herbaria for permission to work with their specimens: Harvard University Herbaria, the Missouri Botanical Garden, the Field Museum of Natural History, and the New York Botanical Garden. We thank Kellie Heckman for help with sequencing, two anonymous reviewers for their comments, and the Donoghue and Near lab groups at Yale University and the Edwards lab group at Brown University for helpful discussions concerning this manuscript. We also thank Patrick Sweeny, David Neill, and the Herbario Nacional del Ecuador, Museo Ecuatoriano de Ciencias Naturales (QCNE) for their efforts in obtaining permits and collecting Viburnum in Ecuador. Funding was provided by the Division of Botany, Peabody Museum of Natural History at Yale University and the National Science Foundation (IOS-0842800).

\section{Authors' contributions}

WLC carried out DNA sequencing and analyses. WLC and MJD designed the study and wrote the manuscript. Both authors read and approved the final manuscript.

Received: 22 December 2011 Accepted: 30 May 2012 Published: 30 May 2012

\section{References}

1. Ford CS, Ayres KL, Toomey N, Haider N, van Alphen Stahl J, Kelly JL, Wikstron N, Hollingsworth PM, Duff RJ, Hoot SB, Cowan RS, Chase MW Wilkinson MJ: Selection of candidate coding DNA barcoding regions for use on land plants. Bot J Linn Soc 2009, 159:1-11.

2. Hollingsworth ML, Clark A, Forrest LL, Richardson J, Pennington RT, Long DG, Cowan R, Chase MW, Gaudeul M, Hollingsworth PM: Selecting barcoding loci for plants: evaluation of seven candidate loci with species-level sampling in three divergent groups of land plants. Mol Ecol Resour 2009, 9:439-457.

3. Kress WJ, Erickson DL: A two-locus global DNA barcode for land plants: the coding $r b c \mathrm{~L}$ gene complements the non-coding $t r n \mathrm{H}-p s b \mathrm{~A}$ spacer region. PLoS One 2007, 6:e508.

4. Fazekas AJ, Burgess KS, Kesanakurti PR, Graham SW, Newmaster SG, Husband BC, Percy DM, Hajibabaei M, Barrett SCH: Multiple multilocus DNA barcodes from the plastid genome discriminate plant species equally well. PLoS One 2008, 3:e2802

5. CBOL Plant Working Group: A DNA barcode for land plants. P Natl Acad Sci USA 2009, 106:12794-12797.

6. Roy S, Tyagi A, Shulka V, Kumar A, Singh UM, Chaudhary LB, Datt B, Bag SK, Singh PK, Nair NK, Husain T, Tuli R: Universal plant DNA barcode loci may not work in complex groups: a case study with Indian Berberis species. PLoS One 2010, 5:e13674.

7. Liu J, Möller M, Gao L, Zhang D, Li D: DNA barcoding for the discrimination of Eurasian yews (Taxus L., Taxaceae) and the discovery of cryptic species. Mol Ecol Resour 2011, 11:89-100.

8. Wang W, Wu Y, Yan Y, Ermakova M, Kerstetter R, Messing J: DNA barcoding of the Lemnaceae, a family of aquatic monocots. BMC Plant Biol 2010, 10:205.

9. Pettengill JB, Neel MC: An evaluation of candidate plant DNA barcodes and assignment methods in diagnosing 29 species in the genus Agalinis (Orobanchaceae). Am J Bot 2010, 97:1381-1406.

10. Starr JR, Naczi RFC, Chouinard BN: Plant DNA barcodes and species resolution in sedges (Carex, Cyperaceae). Mol Ecol Resour 2009, 9:151-163.

11. Newmaster SG, Ragupathy S: Testing plant barcoding in a sister species complex of pantropical Acacia (Mimosoideae, Fabaceae). Mol Ecol Resour 2009, 9:172-180

12. Hollingsworth PM, Graham SW, Little P: Choosing and using a plant DNA barcode. PLoS One 2011, 6:e19254.

13. Kress WJ, Erickson DL: DNA barcodes: genes, genomics, and bioinformatics. P Natl Acad Sci USA 2008, 105:2761-2762.

14. Fazekas AJ, Kesanakurti PR, Burgess KS, Percy DM, Graham SW, Barrett SCH, Newmaster SG, Hajibabaei M, Husband B: Are plant species inherently harder to discriminate than animal species using DNA barcoding markers? Mol Ecol Resour 2009, 9:130-139.

15. Clement WC, Donoghue MJ: Dissolution of Viburnum section Megalotinus (Adoxaceae) of Southeast Asia and its implications for morphological evolution and biogeography. Int J Plant Sci 2011, 172:559-573.
16. DeSalle R, Egan MG, Siddall M: The unholy trinity: taxonomy, species delimitation and DNA barcoding. Philos T Roy Soc B 2005, 360:1905-1916

17. Lahaye R, van der Bank M, Borarin D, Warner J, Pupulin F, Gigot G, Maurin O, Duthoit S, Barraclough TG, Savolainen V: DNA barcoding the floras of biodiversity hotspots. P Natl Acad Sci USA 2008, 105:2923-2928.

18. Kress WJ, Erickson DL, Jones FA, Swenson NG, Perez R, Sanjur O, Bermingham E: Plant DNA barcodes and a community phylogeny of a tropical forest dynamics plot in Panama. P Natl Acad Sci USA 2009, 106:18621-18626.

19. Le Clerc-Blain J, Starr JR, Bull RD, Saarela JM: A regional approach to plant DNA barcoding provides high species resolution of sedges (Carex and Koresia, Cyperaceae) in the Canadian Arctic archipelago. Mol Ecol Resour 2010, 10:69-91.

20. Gonzalez MA, Baraloto C, Engel J, Mori SA, Pétronelli P, Riéra B, Roger A, Thébaud C, Chase J: Identification of Amazonian trees with DNA barcodes. PLoS One 2009, 4:e7483.

21. Dirr MA: Viburnums: flowering shrubs for every season. Portland, Oregon: Timber Press Inc; 2005

22. Egolf DR: Cytological and interspecific hybridization studies in the genus Viburnum. PhD thesis.: Cornell University; 1956.

23. Egolf DR: A cytological study of the genus Viburnum. J Arnold Arb 1962, 43:132-172.

24. Brumbaugh $\mathrm{JH}$, Guard AT: A study of evidences for introgression among Viburnum lentago, $V$. prunifolium, and $V$. rufidulum based on leaf characteristics. Proc Indiana Acad Sci 1956, 66:300.

25. Winkworth RC, Donoghue MJ: Viburnum phylogeny: Evidence from the duplicated nuclear gene GBSSI. Mol Phylogenet Evol 2004, 33:109-126.

26. Winkworth RC, Donoghue MJ: Viburnum phylogeny based on combined molecular data: implications for taxonomy and biogeography. Am J Bot 2005, 92:653-666.

27. Donoghue MJ: Systematic studies in the genus Viburnum. PhD thesis: Harvard University, Department of Organismal and Evolutionary Biology; 1982.

28. Donoghue MJ, Baldwin BG, Li J, Winkworth RC: Viburnum phylogeny based on the chloroplast trnK intron and nuclear ribosomal ITS DNA sequences. Syst Bot 2004, 29:188-198.

29. Kress WJ, Wurdack KJ, Zimmer EA, Weigt LA, Janzen DH: Use of DNA barcodes to identify flowering plants. P Natl Acad Sci USA 2005, 102:83698374.

30. Chen S, Yao H, Han J, Liu C, Song J, Shi L, Zhu Y, Ma X, Gao T, Pang X, Luo $K$, Li Y, Li X, Jia X, Lin Y, Leon C: Validation of the ITS Region as a novel DNA barcode for identifying medicinal plant species. PLoS One 2010, 5: e8613.

31. Gao T, Yao H, Song J, Zhu Y, Liu C, Chen S: Evaluating the feasibility of using candidate DNA barcodes in discriminating species of the large Asteraceae family. BMC Evolutionary Biol 2010, 10:324.

32. Yao H, Song J, Liu C, Luo K, Han J, Li Y, Pang X, Xu H, Zhu Y, Xiao P, Chen S: Use of ITS2 region as the universal DNA barcode for plants and animals. PLoS One 2010, 5:e13102.

33. Hara H: A revision of Caprifoliaceae of Japan with reference to allied plants in other districts and the Adoxaceae. In Ginkgoana, no. 5. Edited by. Tokyo: Academia Scientific Book Inc; 1983.

34. Yang Q-E, Malécot V: Viburnum. In Flora of China, Vol 19. Edited by Wu Z-Y, Raven PH, Hong DY. Beijing and St. Louis: Science Press: Beijing and Missouri Botanical Garden Press; 2011:570-611.

35. Haines A: Flora Novae Angliae: A manual for the identification of native and naturalized higher vascular plants of New England. New Haven: Yale University Press; 2011.

36. Wurdack K, Hoffmann P, Samuel R, de Bruijn A, van der Bank M, Chase M: Molecular phylogenetic analysis of Phyllanthaceae (Phyllanthoideae pro parte, Euphorbiaceae sensu lato) using plastid rbcL DNA sequences. Am J Bot 2004, 91:1882-1900.

37. Edgar RC: MUSCLE: multiple sequence alignment with high accuracy and high throughput. Nucleic Acids Res 2004, 32:1792-1797.

38. Nylander JAA: MrModeltest Version 2. Evolutionary Biology Centre, Uppsala University:: ; 2004. http://www.abc.se/ nylander/mrmodeltest2/ mrmodeltest $2 . h t m l$.

39. Huelsenbeck JP, Ronquist FR: MrBayes: Bayesian inference of phylogeny. Biometrics 2001, 17:754-755.

40. Rambaut A, Drummond AJ: Tracer v1.5.: ; http://beast.bio.ed.ac.uk/software/ tracer. 
41. Clement M, Posada D, Crandall D: TCS: a computer program to estimate gene genealogies. Mol Ecol 2000, 9:1657-1660.

42. Swofford DL: PAUP*: phylogenetic analysis using parsimony (*and other methods). Version 4. Sunderland, MA: Sinauer; 2002.

43. R Development Core Team: $R$ : A language and environment for statistical computing.: ; http://www.R-project.org/.

44. Meyer CP, Paulay G: DNA barcoding: error rates based on comprehensive sampling. PLOS Biol 2005, 3:e422.

45. Whitlock BA, Hale AM, Groff PA: Intraspecific inversions pose a challenge for the trnH-psbA plant DNA barcode. PLoS One 2010, 5:E11533.

46. Hollingsworth PM: Refining the DNA barcode for land plants. P Natl Acad Sci USA 2011, 108:19451-19452.

47. China Plant BOL Group: Comparative analysis of a large dataset indicates that internal transcribed spacer (ITS) should be incorporated into the core barcode for seed plants. PNAS 2011, 108:19641-19646.

48. Ren B-Q, Xiang X-G, Chen Z-D: Species identification of Alnus (Betulaceae) using nrDNA and cpDNA genetic markers. Mol Ecol Resour 2010, 10:594605 .

49. Gu J, Su J-X, Lin R-Z, Li R-Q, Xiao P-G: Testing four proposed barcoding markers for the identification of species within Ligustrum L. (Oleaceae). J Syst Evol 2011, 49:213-224.

50. Xiang X-G, Zhang J-B, Lu A-M, Li R-Q: Molecular identification of species in Juglandaceae: a tiered method. J Syst Evol 2011, 49:252-260.

51. Piredda R, Simeone MC, Attimonelli M, Bellarosa R, Schirone C: Prospects of barcoding the Italian wild dendroflora: oaks reveal severe limitations to tracking species identity. Mol Ecol Resour 2011, 11:72-83.

52. Smith SA, Donoghue MJ: Rates of molecular evolution are linked to life history in flowering plants. Science 2008, 322:86-89.

53. Nicolalde-Morejón F, Vergara-Silva F, González-Astorga J, Stevenson DW, Vovides AP, Sosa V: A character-based approach in the Mexican cycads supports diverse multigene combinations for DNA barcoding. Cladistics 2010, 27:150-164.

54. Ran J-H, Wang P-P, Zhao H-J, Wang X-Q: A test of seven candidate barcode regions from the plastome in Picea (Pinaceae). J Integr Plant Biol 2010, 52:1109-1126.

55. Newmaster SG, Fazekas AJ, Steeves RAD, Janovec J: Testing candidate plant barcode regions in the Myristicaceae. Mol Ecol Resour 2008, 8:480490

56. Morton CV: The Mexican and Central American species of Viburnum. Contrib USA Natl Herb 1933, 26:339-366.

57. Killip EP, Smith AC: The South American species of Viburnum. B Torrey Bot Club 1930, 57:245-258

58. Moore BR, Donoghue MJ: Correlates of diversification in the plant clade Dipsacales: Geographic movement and evolutionary innovations. Am Nat 2007, 170:\$29-\$55.

59. Moore BR, Donoghue MJ: A Bayesian approach for evaluating the impact of historical events on rates of diversification. P Natl Acad Sci USA 2009, 106:4307-4312.

doi:10.1186/1471-2148-12-73

Cite this article as: Clement and Donoghue: Barcoding success as a function of phylogenetic relatedness in Viburnum, a clade of woody angiosperms. BMC Evolutionary Biology 2012 12:73.

\section{Submit your next manuscript to BioMed Central and take full advantage of:}

- Convenient online submission

- Thorough peer review

- No space constraints or color figure charges

- Immediate publication on acceptance

- Inclusion in PubMed, CAS, Scopus and Google Scholar

- Research which is freely available for redistribution

Submit your manuscript at www.biomedcentral.com/submit
Biomed Central 